\title{
Heterogeneity in disease risk induces falling vaccine protection with rising disease incidence
}

\author{
Alessandro Margheri * \\ Centro de Matemática Aplicações Fundamentais e Investigação Operacional \\ Faculdade de Ciências, Universidade de Lisboa \\ Campo Grande, Edificio C6, piso 2 \\ 1749-016, Lisboa, Portugal \\ e-mail: amargheri@fc.ul.pt \\ Carlota Rebelo ${ }^{\dagger}$ \\ Centro de Matemática Aplicações Fundamentais e Investigação Operacional \\ Faculdade de Ciências, Universidade de Lisboa \\ Campo Grande, Edificio C6, piso 2 \\ 1749-016, Lisboa, Portugal \\ e-mail: mcgoncalves@fc.ul.pt

\section{Gabriela M. Gomes $\ddagger$} \\ CIBIO-InBIO, Centro de Investigação em Biodiversidade e Recursos Genéticos \\ Universidade do Porto, Porto, Portugal \\ Instituto de Matemática e Estatística, Universidade de São Paulo, São Paulo, Brazil \\ Liverpool School of Tropical Medicine, Liverpool United Kingdom. \\ e-mail: gabriela.gomes@lstmed.ac.uk
}

April 14, 2016

\footnotetext{
* Supported by Fundação para a Ciência e aTecnologia, UID/MAT/04561/2013.

${ }^{\dagger}$ Supported by Fundação para a Ciência e a Tecnologia, UID/MAT/04561/2013.

‡Supported by Fundação para a Ciência e a Tecnologia (FCT) and Coordenação de Aperfeiçoamento de Pessoal de Nível Superior (CAPES).
} 


\begin{abstract}
This paper is concerned with the analysis of phase 3 vaccine trials. In a randomised controlled trial, a representative sample of a population is given a vaccine and a matched sample is given a placebo. These individuals are followed for a stipulated length of time, while infection (or disease) occurrences are registered. Vaccine efficacy is then calculated to measure the reduction in disease rate (or risk) attributed to the vaccine. Seemingly very reasonable, this procedure often results in the most disparate estimates when conducted in different parts of the world. Here we argue that this is due to cohort selection acting on the trial participants as follows. The more susceptible individuals are infected first, leaving behind a pool whose mean susceptibility decreases over time. As a result infection rates decrease, and this effect is stronger in the control group provided that the vaccine reduces susceptibility. Therefore, any direct measure of vaccine efficacy is expected to decrease as the trial progresses, and this happens faster in settings where the intensity of pathogen exposure is higher. We propose an analytical scheme that takes this phenomenon into account while estimating efficacy more consistently across settings. We provide analytical results concerning the dependence of vaccine efficacy on the intensity of pathogen exposure as well as on the mean and variance of the distribution of disease risk.
\end{abstract}

\title{
1 Introduction
}

Vaccine development is a lengthy process, lasting in the order of 10-15 years. Typically it includes an initial stage, of basic laboratory research and preclinical animal studies, to identify formulations with the ability to elicit an effective immune response. Some products are then approved to proceed to clinical studies in human subjects. A series of vaccine trials of increasing scale is then performed: phase 1 trials involve 20-80 subjects to assess whether the vaccine is safe in humans and what immune response it evokes; phase 2 trials involve several hundred individuals to assess the efficacy of the vaccine against artificial infection and clinical disease; phase 3 trials involve thousands of subjects across several sites to evaluate efficacy under natural disease condition.

Phase 3 trials are essentially conducted according to randomised controlled designs, whereby a representative sample of a population receives a vaccine and a matched sample is given a placebo, in a highly regulated manner. These individuals are followed for a stipulated length of time, while infection (or disease) occurrences are registered. Vaccine efficacy is then calculated to measure the reduction in disease rate (or risk) attributed to the 
vaccine $[4,13]$. This often results in a disparity of results when trials are conducted in different settings $[5,6,11]$, with a consistent tendency for lower vaccine protection in regions where the intensity of transmission is higher. Here we examine the mathematical basis for such trend.

We argue that this is due to a selection process acting on the cohort of trial participants as follows. The more susceptible individuals are infected first, leaving behind a pool whose mean susceptibility decreases with time. As a result infection rates decrease over time, and this effect is stronger in the control group provided that the vaccine reduces susceptibility. Therefore, any measure of vaccine efficacy is expected to decrease as the trial progresses, unless heterogeneity is accounted for, and this happens faster in settings where exposure to the pathogen is higher.

The paper is organised as follows. Section 2 introduces a minimal model to provide context to current vaccine trial procedures. Section 3 shows the model's inadequacy to consistently fit efficacy estimates for vaccines against tuberculosis (BCG) and malaria (RTS,S), as measured in different parts of the world. We reason that the discrepancies may be due to individual heterogeneity in disease risk and, in Section 4, reformulate the model to consider that disease risk follows a gamma distribution. Efficacy curves generated numerically with the new model show a tendency for decreasing vaccine protection with increasing intensity of exposure. In Section 5, we propose an alternative measure of vaccine efficacy, which might work more consistently across settings.

For simplicity, we assume that infection coincides with disease and use the two terms interchangeably. Analogously, we use non-infected and healthy interchangeably to refer to individuals who are free of the disease under study. Besides being in the interest of simplicity, this convention also helps convey that the results presented here are not specific to infectious diseases, but are more generally applicable to any response to a stimulus that can be exerted in different intensities.

\section{Minimal model for a vaccine trial}

Any analysis of a vaccine trial must compare some measure of disease occurrence in two groups of the population that are differentiated by whether or not they have received the vaccine, here named the vaccine group and the control group. To model disease occurrences in the context of a trial it is reasonable to assume that the intensity of exposure remains constant given that the vaccine group represents only a small fraction of the population 
and, therefore, eventual reductions in this group do not significantly affect the prevalence of infection overall. A minimal model for the control group can then be formalised by a system of linear differential equations as

$$
\begin{aligned}
\frac{\mathrm{d} S_{c}}{\mathrm{~d} t} & =-\lambda S_{c}+\gamma\left(1-S_{c}\right), \\
\frac{\mathrm{d} I_{c}}{\mathrm{~d} t} & =\lambda S_{c},
\end{aligned}
$$

where $\lambda$ is the per capita disease rate, also termed force of infection, which applies to healthy individuals (proportion $S_{c}$ of the control group), and $\gamma$ is the rate of recovery, which applies to those who have the disease (proportion $1-S_{c}$ of the control group), and time is measured in years. Note that $I_{c}$ is not the proportion of the population who has the disease at any given time (as typical in mathematical epidemiology textbooks [1]) but rather the cumulative number of disease occurrences per person, in this case belonging to the control group. The model for the vaccine group is analogously written as

$$
\begin{aligned}
\frac{\mathrm{d} S_{v}}{\mathrm{~d} t} & =-\sigma \lambda S_{v}+\gamma\left(1-S_{v}\right) \\
\frac{\mathrm{d} I_{v}}{\mathrm{~d} t} & =\sigma \lambda S_{v}
\end{aligned}
$$

where $\sigma$ is the reduction factor applied to the per capita disease rate due to vaccination, $S_{v}$ represents the healthy proportion of the vaccine group and $I_{v}$ the cumulative number of disease occurrences per person, in the vaccine group. Note that making the recovery rate equal to zero $(\gamma=$ 0 ) may be representative of an infectious disease which is either a longlasting condition or induces long-lasting immunity, or a study design where individuals are permanently removed from the trial once they acquire the disease. Conversely, $\gamma>0$ represents a scenario where individuals regain their susceptibility and are placed back in the trial upon recovery.

The equations for the rates of change in control individuals $(1,2)$, and their vaccine group analogues $(3,4)$, can be solved analytically. Given that all individuals are non-infected at the beginning of the trial $\left(S_{c}(0)=S_{v}(0)=1\right.$ 
and $\left.I_{c}(0)=I_{v}(0)=0\right)$, we have

$$
\begin{aligned}
S_{c}(t) & =\frac{\gamma}{\lambda+\gamma}+\frac{\lambda}{\lambda+\gamma} \mathrm{e}^{-(\lambda+\gamma) t}, \\
I_{c}(t) & =\frac{\lambda \gamma}{\lambda+\gamma} t+\frac{\lambda^{2}}{(\lambda+\gamma)^{2}}\left[1-\mathrm{e}^{-(\lambda+\gamma) t}\right], \\
S_{v}(t) & =\frac{\gamma}{\sigma \lambda+\gamma}+\frac{\sigma \lambda}{\sigma \lambda+\gamma} \mathrm{e}^{-(\sigma \lambda+\gamma) t}, \\
I_{v}(t) & =\frac{\sigma \lambda \gamma}{\sigma \lambda+\gamma} t+\frac{(\sigma \lambda)^{2}}{(\sigma \lambda+\gamma)^{2}}\left[1-\mathrm{e}^{-(\sigma \lambda+\gamma) t}\right] .
\end{aligned}
$$

In practice, as the trial is conducted, researchers record events of disease acquisition and recovery. This information is then used to calculate the disease rate, also called disease incidence, defined as the number of cases per person-years at risk. In terms of our models, the instantaneous rates are expressed as

$$
\begin{aligned}
& r_{c}(t)=\lambda, \\
& r_{v}(t)=\sigma \lambda,
\end{aligned}
$$

in this case constant in time, as previous noted by Smith et al [13] and further by Halloran et al [4]. Vaccine efficacy is then calculated as the proportional reduction in incidence due to vaccination, this is

$$
V E_{1}=\frac{r_{c}-r_{v}}{r_{c}}=1-\frac{r_{v}}{r_{c}}=1-\sigma,
$$

which is independent of baseline disease rate, $\lambda$. Alternatively, another measure commonly used in clinical trials is the disease risk, which reports the cumulative number of disease occurrences per person at risk. Considering a trial of duration $T$, this gives

$$
V E_{2}=1-\frac{I_{v}(T)}{I_{c}(T)}
$$

which appears to be a decreasing function of $\lambda$ due to depletion of susceptibles, occuring faster in the control group than in those vaccinated given that vaccine reduces susceptibility somewhat (Figure 1A,B).

Figure 1 illustrates the two formulations of vaccine efficacy. The ratebased measure $V E_{1}$ is, as expected, constant over $\lambda$ (Figure 1B, horizontal dotted line in black), and independent of the recovery rate $\gamma$. The riskbased measure $V E_{2}$ decreases with $\lambda$ with a slope that is attenuated by $\gamma$ 
(Figure $1 \mathrm{~B}, \gamma=0$ in blue and $\gamma=12$ in red). Figure $1 \mathrm{~A}$ shows cumulative disease occurrences per 100 persons in a trial of one-year duration (blue and red refer to $\gamma$ as before, while solid and dash-dotted curves correspond to control and vaccine groups, $I_{c}$ and $I_{v}$, respectively). The black lines in Figure $1 \mathrm{~A}$ are shown to indicate what the cumulative disease occurrences would have been had the susceptible pool not been subject to depletion by the disease. Equivalently, the black lines coincide with the disease rates, in this case per 100 person-years.
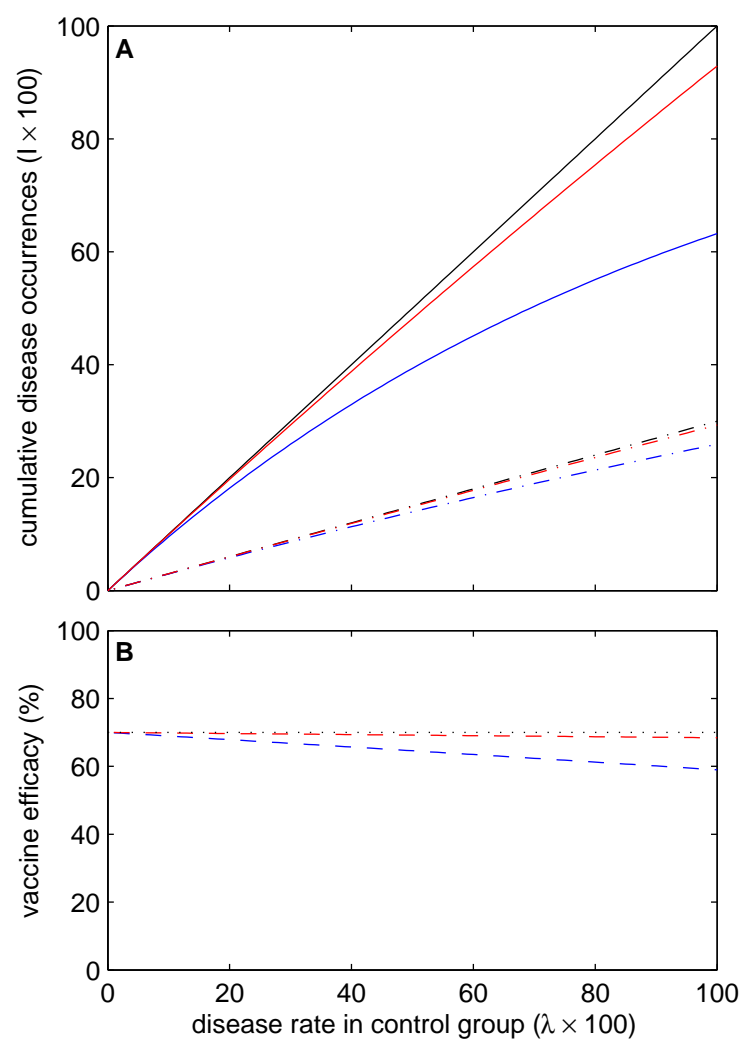

Figure 1: Two formulations of vaccine efficacy. A, Coloured curves represent cumulative disease occurrences per 100 persons in a trial of one-year duration (solid and dash-dotted curves correspond to control and vaccine groups, $I_{c}$ and $I_{v}$, respectively). Black lines indicate disease rates. B, Rate-based $V E_{1}$ in black, and risk-based $V E_{2}$ in colour. Blue and red refer to $\gamma=0$ and $\gamma=12$, respectively. 


\section{Data from randomised clinical trials}

We have extracted, from the literature, vaccine efficacy estimates on tuberculosis (Table 1) and malaria (Table 2). Table 1 shows data on neonatal vaccination with the Bacille Calmette-Guérin (BCG) vaccine against pulmonary tuberculosis (5 studies from a systematic literature review [6]), and Table 2 lists data on the RTS,S vaccine against clinical malaria (11 sites from a multi-center clinical trial [11]). Both datasets are structured by study site and plotted in Figure 2.

The dotted lines in Figure 2 represent $1-\sigma$, according to the rate-based measure $V E_{1}$, which here we set at the level of the highest efficacy estimate as a first approximation. As changing parameter $\sigma$ basically moves these horizontal lines up and down, it is evident that this cannot consistently fit the data.

The dashed lines represent the risk-based measures $V E_{2}$, for $\gamma=0$ in the case of tuberculosis and $\gamma=12$ in the case of malaria. Although these show a downward trend as in the trial measurements, it appears so shallow that the slope is hardly detectable when plotted in a scale comparable with the data.

Although both $V E_{1}$ and $V E_{2}$ are approximations for the vaccine efficacy, in theory one is always more appropriate than the other depending on the vaccine mode of action or study design [13]. In the particular datasets used here, $V E_{1}$ should be more adequate since both studies are based on ratebased measures of disease. In any case, the two measures perform very similarly when confronted with the data.

Since neither of these simple measures is able to fit the data at the global scale, the topic of variable vaccine efficacy continues to motivate field studies to seek explanations for the observed discrepancies. Such projects tend to be very large and expensive, and are almost invariably based on a biological rationale for the immunological response to the vaccine to be higher in some populations than in others. Here we show, mathematically, that this is not necessarily the case. Central to our argument is that individual heterogeneity is present in every setting and the mean field approximations described above are poor representations of what is actually being measured in the field. Moreover, we argue that randomised study designs, considered the gold standard for clinical trials, deliberately selected heterogeneous groups promoting the discrepancies under discussion. 
Table 1: BCG vaccine protection against pulmonary tuberculosis [11].

\begin{tabular}{lll}
\hline Site, Country & $\begin{array}{l}\text { Incidence } \\
\text { (per 100 person-years) }\end{array}$ & $\begin{array}{l}\text { Vaccine efficacy } \\
\mathbf{\%}(\mathbf{9 5 \%} \mathbf{C I})\end{array}$ \\
\hline Saskatchewan, Canada & 0.7 & $80(31,94)$ \\
Chicago (hospital), USA & 0.3 & $66(40,81)$ \\
Chicago (households), USA & 0.8 & $72(-2,92)$ \\
Turtle and Rosebud, USA & 1.0 & $60(-25,87)$ \\
Bombay, India & 3.8 & $38(-7,65)$ \\
Overall & - & $59(42,71)$ \\
\hline
\end{tabular}

Table 2: RTS,S vaccine protection against clinical malaria [6].

\begin{tabular}{lll}
\hline Site, Country & $\begin{array}{l}\text { Incidence } \\
\text { (per } \mathbf{1 0 0} \text { person-years) }\end{array}$ & $\begin{array}{l}\text { Vaccine efficacy } \\
\mathbf{\%}(\mathbf{9 5 \%} \mathbf{C I})\end{array}$ \\
\hline Kilifi, Kenya & 10.4 & $66.0(37.5,81.5)$ \\
Korogwe, Tanzania & 5.9 & $52.0(26.2,68.8)$ \\
Manhiça, Mozambique & 8.5 & $33.3(7.1,52.1)$ \\
Lambaréné, Gabon & 17.7 & $36.1(10.8,54.1)$ \\
Bagamoyo, Tanzania & 13.2 & $37.5(13.5,54.9)$ \\
Lilongwe, Malawi & 16.6 & $33.5(8.2,51.8)$ \\
Agogo, Ghana & 85.8 & $31.1(13.3,45.2)$ \\
Kombewa, Kenya & 48.4 & $27.1(12.9,38.9)$ \\
Kintampo, Ghana & 66.4 & $25.9(15.0,35.4)$ \\
Niamoro, Burkina Faso & 120.7 & $17.7(7.0,27.2)$ \\
Siaya, Kenya & 139.0 & $20.2(7.4,31.3)$ \\
Overall & - & $28.2(23.3,32.9)$ \\
\hline
\end{tabular}

\section{Introducing heterogeneity in disease risk}

Individual heterogeneity in disease risk can be due to biological factors intrinsic to each individual, such as susceptibility to infection [7] in the case of infectious diseases, or to extrinsic factors affecting exposure to the infectious agent, such as contact dynamics [8]. In reality, many factors (intrinsic and extrinsic) combine to determine the risk distribution, $q(x)$, a probability density function describing individual risks. In this setting, $q(x) \mathrm{d} x$ approximates the fraction of the population of type $x$.

The model for the control group in a heterogeneous population is then written as an infinite system of linear differential equations

$$
\begin{aligned}
\frac{\mathrm{d} S_{c}}{\mathrm{~d} t}(x) & =-\lambda x S_{c}(x)+\gamma\left(q(x)-S_{c}(x)\right) \\
\frac{\mathrm{d} I_{c}}{\mathrm{~d} t}(x) & =\lambda x S_{c}(x)
\end{aligned}
$$



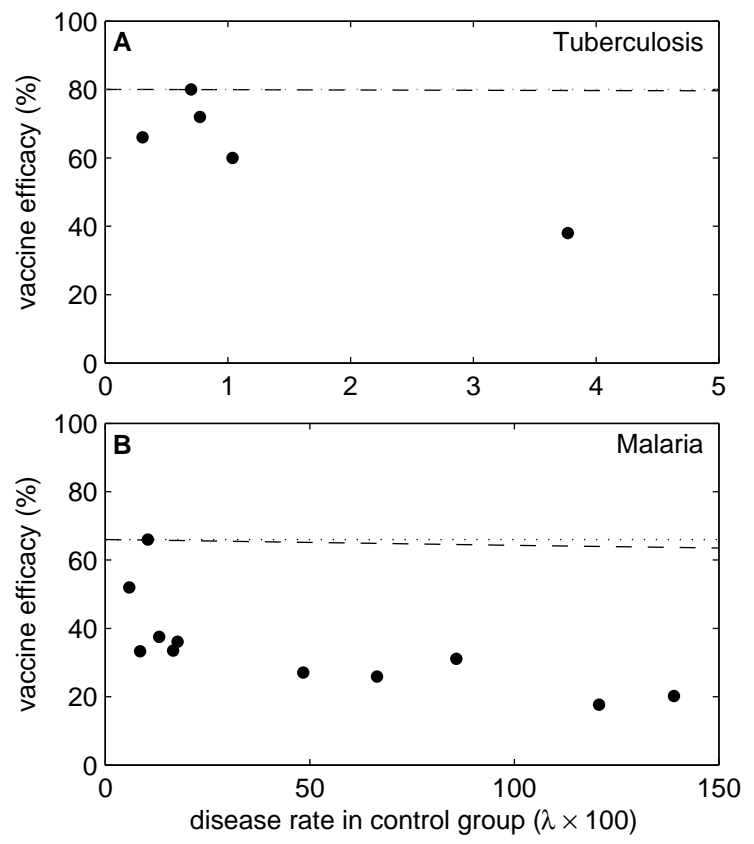

Figure 2: Vaccine efficacy data from randomised controlled trials. A, Tuberculosis [6]. B, Malaria [11]. Dotted and dashed lines represent $V E_{1}$ and $V E_{2}$, respectively.

and, analogously, for the vaccine group we have

$$
\begin{aligned}
\frac{\mathrm{d} S_{v}}{\mathrm{~d} t}(x) & =-\sigma \lambda x S_{v}(x)+\gamma\left(q(x)-S_{v}(x)\right), \\
\frac{\mathrm{d} I_{v}}{\mathrm{~d} t}(x) & =\sigma \lambda x S_{v}(x) .
\end{aligned}
$$

The model solutions become

$$
\begin{aligned}
S_{c}(x, t) & =\frac{\gamma q(x)}{\lambda x+\gamma}+\frac{\lambda x q(x)}{\lambda x+\gamma} \mathrm{e}^{-(\lambda x+\gamma) t}, \\
I_{c}(x, t) & =\frac{\lambda x \gamma q(x)}{\lambda x+\gamma} t+\frac{(\lambda x)^{2} q(x)}{(\lambda x+\gamma)^{2}}\left[1-\mathrm{e}^{-(\lambda x+\gamma) t}\right], \\
S_{v}(x, t) & =\frac{\gamma q(x)}{\sigma \lambda x+\gamma}+\frac{\sigma \lambda x q(x)}{\sigma \lambda x+\gamma} \mathrm{e}^{-(\sigma \lambda x+\gamma) t}, \\
I_{v}(x, t) & =\frac{\sigma \lambda x \gamma q(x)}{\sigma \lambda x+\gamma} t+\frac{(\sigma \lambda x)^{2} q(x)}{(\sigma \lambda x+\gamma)^{2}}\left[1-\mathrm{e}^{-(\sigma \lambda x+\gamma) t}\right] .
\end{aligned}
$$


In what follows, we consider a risk distribution $q(x)$ with finite mean $\mu=$ $E[x]=\int_{0}^{+\infty} x q(x) \mathrm{d} x$ and finite variance $\operatorname{Var}=V[x]=\int_{0}^{+\infty}(x-\mu)^{2} q(x) \mathrm{d} x$.

\subsection{Rate-based analysis with heterogeneity}

From system (13-16) and solutions (17-20), the instantaneous infection rates are

$$
\begin{aligned}
& r_{c}(t)=\frac{\int_{0}^{\infty} \lambda x S_{c}(x, t) \mathrm{d} x}{\int_{0}^{\infty} S_{c}(x, t) \mathrm{d} x}=\frac{\int_{0}^{\infty} \frac{\lambda x q(x)}{\lambda x+\gamma}\left[\gamma+\lambda x \mathrm{e}^{-(\lambda x+\gamma) t}\right] \mathrm{d} x}{\int_{0}^{\infty} \frac{q(x)}{\lambda x+\gamma}\left[\gamma+\lambda x \mathrm{e}^{-(\lambda x+\gamma) t}\right] \mathrm{d} x} \\
& r_{v}(t)=\frac{\int_{0}^{\infty} \sigma \lambda x S_{v}(x, t) \mathrm{d} x}{\int_{0}^{\infty} S_{v}(x, t) \mathrm{d} x}=\frac{\int_{0}^{\infty} \frac{\sigma \lambda x q(x)}{\sigma \lambda x+\gamma}\left[\gamma+\sigma \lambda x \mathrm{e}^{-(\sigma \lambda x+\gamma) t}\right] \mathrm{d} x}{\int_{0}^{\infty} \frac{q(x)}{\sigma \lambda x+\gamma}\left[\gamma+\sigma \lambda x \mathrm{e}^{-(\sigma \lambda x+\gamma) t}\right] \mathrm{d} x}
\end{aligned}
$$

which intuitively are decreasing functions of time due to cohort selection for reduced susceptibility $[9,14,15]$. As long as the vaccine is protective (i.e. $\sigma<1$ ), this effect is more pronounced in $r_{c}(t)$ than in $r_{v}(t)$ as individuals in the control group are infected faster. Consequently, the ratio of disease rates in vaccinated over control groups increases, and vaccine efficacy decreases as the trial progresses. Finally, the magnitude of this effect increases with the force of infection.

In what follows we analyse these trends more formally in comparison with the data presented in the previous section. Emphasising its dependence on $\sigma, \lambda$ and $q(x)$, the rate-based vaccine efficacy expected from a trial of duration $T$ in a heterogeneous population is written as

$$
V E_{1}(\sigma, \lambda, q, T)=1-\frac{\frac{\int_{0}^{T} \int_{0}^{\infty} \sigma \lambda x S_{v}(x, t) \mathrm{d} x \mathrm{~d} t}{\int_{0}^{T} \int_{0}^{\infty} S_{v}(x, t) \mathrm{d} x \mathrm{~d} t}}{\frac{\int_{0}^{T} \int_{0}^{\infty} \lambda x S_{c}(x, t) \mathrm{d} x \mathrm{~d} t}{\int_{0}^{T} \int_{0}^{\infty} S_{c}(x, t) \mathrm{d} x \mathrm{~d} t}},
$$

whose tendency to decrease with $\lambda$ is illustrated by the plots in Figure $3 \mathrm{~A}, \mathrm{C}$ adopting for $q(x)$ gamma distributions with different variances. In the following result we prove this tendency for small positive values of $\lambda$ for any probability distribution $q$. The homogeneous case considered in Section 2 is retrieved when $q$ is a delta distribution.

Theorem 4.1 The function $V E_{1}(\sigma, \lambda, q, T)$ satisfies

$$
V E_{1}(\sigma, 0, q, T)=1-\sigma, \quad \frac{\partial V E_{1}}{\partial \lambda}(\sigma, 0, q, T)=\left\{\begin{array}{ll}
\sigma(\sigma-1) \frac{C_{\gamma, T}}{\gamma T} \frac{V a r}{\mu}, & \text { if } \gamma>0 \\
\sigma(\sigma-1) \frac{T}{2} \frac{V a r}{\mu}, & \text { if } \gamma=0
\end{array},\right.
$$


where $C_{\gamma, T}=\frac{e^{-\gamma T}-1}{\gamma}+T>0$.

Proof. We notice first that the factor $\lambda$ appearing in the numerators of $r_{v}(t)$ and $r_{c}(t)$ cancels out in equation (23). To simplify the notations and emphasize the variables relevant for our calculations, we rewrite (23) after the cancellation of the factor $\lambda$ as

$$
V E_{1}(\sigma, \lambda)=1-\frac{\frac{A(\sigma, \lambda)}{B(\sigma, \lambda)}}{\frac{A(1, \lambda)}{B(1, \lambda)}},
$$

where, of course, we set

$$
A(\sigma, \lambda)=\int_{0}^{T} \int_{0}^{\infty} \sigma x S_{v}(x, t) \mathrm{d} x \mathrm{~d} t
$$

and $B(\sigma, \lambda)=\int_{0}^{T} \int_{0}^{\infty} S_{v}(x, t) \mathrm{d} x \mathrm{~d} t$. Since for any $\left.\left.\sigma \in\right] 0,1\right]$ we have $A(\sigma, 0)=$ $\sigma T \mu$, and $B(\sigma, 0)=T$, the first formula in (24) follows immediately. For any values of $\sigma$ and $\lambda$, the four functions $\sigma x S_{v}(x, t), x S_{c}(x, t), \sigma S_{v}(x, t)$ and $S_{c}(x, t)$ are dominated on $[0, T] \times[0,+\infty[$ by the integrable function $(x+$ 1) $q(x)$. Then, we can interchange the order of integration in the integrals of formula (23). Moreover, fixed a compact set $K$ in $] 0,+\infty[$, for any $\lambda \in K$ there exist constants $C_{K}, D_{K}>0$ such that the derivatives with respect to $\lambda$ of all these four functions are dominated on $[0, T] \times[0,+\infty[$ by the integrable function $\left(C_{K}+D_{K} x\right) q(x)$. As a consequence, we can compute the $\lambda$ derivatives of the integrals in (25) by differentiating under the integral sign, and get the second formula in (24) directly by the quotient rule for derivatives taking into account that

$$
\frac{\partial A}{\partial \lambda}(\sigma, 0)=-\frac{\sigma^{2}}{\gamma} C_{\gamma, T}\left(\operatorname{Var}+\mu^{2}\right), \frac{\partial B}{\partial \lambda}(\sigma, 0)=-\frac{\sigma}{\gamma} C_{\gamma, T} \mu,
$$

if $\gamma>0$, whereas for $\gamma=0$ analogous formulas hold replacing $\frac{C_{\gamma, T}}{\gamma}$ with $\frac{T^{2}}{2}$.

We notice that, consistently with the plots in Figure 3A,C, given two distributions $q_{1}$ and $q_{2}$ with the same mean value $\mu$ and such that $q_{2}$ has a greater variance than $q_{1}$, there exists a suitable interval $\left.\left.J=\right] 0, \bar{\lambda}\right]$ such that the functions $\lambda \rightarrow V E_{1}\left(\sigma, \lambda, q_{i}, T\right), \quad i=1,2$, are decreasing in $J$ and are ordered as

$$
V E_{1}\left(\sigma, \lambda, q_{1}, T\right)>V E_{1}\left(\sigma, \lambda, q_{2}, T\right), \quad \lambda \in J .
$$


The natural question is whether the negative correlation with the variance of $q$ still holds as $\lambda$ increases. To address this question analytically for the previous model appears a very difficult task, even for a special choice of $q$, and is beyond the scope of this paper. Meanwhile, the numerical solutions in Figure 3A,C indicate that the trend presists as $\lambda$ is increased further, at least within the range that is relevant for the data.

\subsection{Risk-based analysis with heterogeneity}

The case of risk-based analysis is more amenable to mathematical treatment and we are able to provide proofs concerning vaccine efficacy trends more globally. In the following we give some results about the impact of both variance in disease risk and overall intensity of exposure on the vaccine efficacy calculated from risk ratios. Emphasising its dependence on $\sigma, \lambda$ and $q(x)$, we express the risk-based vaccine efficacy as

$$
V E_{2}(\sigma, \lambda, q, T)=1-\frac{\int_{0}^{\infty} I_{v}(x, T) \mathrm{d} x}{\int_{0}^{\infty} I_{c}(x, T) \mathrm{d} x} .
$$

Our next result is the analogue of Theorem 4.1 for the risk-based model. The homogeneous case considered in Section 2 is again obtained when $q$ is a delta distribution.

Theorem 4.2 The function $V E_{2}(\sigma, \lambda, q, T)$ satisfies

$V E_{2}(\sigma, 0, q, T)=1-\sigma, \frac{\partial V E_{2}}{\partial \lambda}(\sigma, 0, q, T)=\left\{\begin{array}{ll}\sigma(\sigma-1) \frac{C_{\gamma, T}}{\gamma T}\left(\frac{V a r}{\mu}+\mu\right), & \text { if } \gamma>0 \\ \sigma(\sigma-1) \frac{T}{2}\left(\frac{V a r}{\mu}+\mu\right), & \text { if } \gamma=0\end{array}\right.$,

where $C_{\gamma, T}>0$ is as in Theorem 4.1.

Proof. The proof is a straightforward consequence of the identity

$$
\frac{\int_{0}^{\infty} I_{v}(x, T) \mathrm{d} x}{\int_{0}^{\infty} I_{c}(x, T) \mathrm{d} x}=\frac{A(\sigma, \lambda)}{A(1, \lambda)}
$$

where $A(\sigma, \lambda)$ is defined in (26), and of the first equation in (27), in which, when $\gamma=0$,one has to replace $\frac{C_{\gamma, T}}{\gamma}$ with $\frac{T^{2}}{2}$.

Notice that Theorem 4.2 shows that the trends for $V E_{2}$ are the same as those established in Theorem 4.1 for $V E_{1}$. Therefore, all the comments made above about the behaviour of $V E_{1}$ for small values of $\lambda$ may be repeated 
for $V E_{2}$. However, in this case, the question of whether or not the negative correlation with the variance of $q$ still holds as $\lambda$ increases may be answered analytically, considering the scenario without reinfection $(\gamma=0)$ in the special case of the gamma distribution. Since the results we present below are of qualitative type and hold for $\lambda$ varying in a suitable interval, without loss of generality, in what follows we assume $T=1$ and no longer write $T$ as an argument of $V E_{2}$. The model without reinfection takes the form

$$
V E_{2}(\sigma, \lambda, q)=1-\frac{1-\int_{0}^{\infty} q(x) \mathrm{e}^{-\sigma \lambda x} \mathrm{~d} x}{1-\int_{0}^{\infty} q(x) \mathrm{e}^{-\lambda x} \mathrm{~d} x} .
$$

From now on we consider that the individual risk is a gamma distributed random variable with probability density function given by

$$
q(x, k, \theta)=\frac{x^{k-1} \mathrm{e}^{-\frac{x}{\theta}}}{\theta^{k} \Gamma(k)},
$$

where $\theta$ and $k$ are positive constants and $\Gamma(\cdot)$ denotes the Gamma function.

We recall that if $x$ is a random variable which is gamma distributed then the mean value of $x$ is $\mu=E[x]=k \theta$ and its variance is $V[x]=k \theta^{2}=\mu \theta$.

Taking into account the expression of the Laplace transform of the probability density function of the gamma distribution and setting with a slight abuse of notation $V E_{2}(\sigma, \lambda, \mu, \theta)=V E_{2}(\sigma, \lambda, q(\cdot, \mu, \theta))$ we obtain

$$
V E_{2}(\sigma, \lambda, \mu, \theta)=1-\frac{1-(1+\sigma \theta \lambda)^{-\frac{\mu}{\theta}}}{1-(1+\theta \lambda)^{-\frac{\mu}{\theta}}} .
$$

The following results give a fairly detailed qualitative description of global behaviour of the vaccine efficacy when the disease risk is gamma distributed. For this special case, they complement Theorem 4.2, which although considering a general distribution was restricted to small positive values of $\lambda$. In particular, from Theorem 4.3 below we see that the length of the $\lambda$ interval on which we can guarantee that the vaccine efficacy correlates negatively with the variance of the distribution is a function of the mean and variance. Actually, in Remark 1 we show that, for fixed mean of the gamma distribution, the larger the variance the larger such $\lambda$ interval. Interestingly, we find that the negative correlation between vaccine efficacy and variance does not hold when transmission rates are sufficiently high; whatever the value of the relative risk of infection in vaccinated vs unvaccinated individuals $(\sigma)$, the efficacy curves cross when $\lambda$ in represented on a suitably large scale (see Corollary 4.1). 
Theorem 4.3 The function $\lambda \rightarrow V E_{2}(\sigma, \lambda, \mu, \theta)$ is strictly decreasing in $\left[0,+\infty[\right.$ for any $(\sigma, \mu, \theta) \in] 0,1\left[\times \mathbb{R}^{+} \times \mathbb{R}^{+}\right.$. Also

i) For any $(\mu, \theta) \in \mathbb{R}^{+} \times \mathbb{R}^{+}$, there exists a positive $\lambda=\lambda(\mu, \theta)$ such that

$$
\left.\left.\frac{\partial V E_{2}}{\partial \theta}(\sigma, \lambda, \mu, \theta)<0, \quad \text { for each } \lambda \in\right] 0, \lambda(\mu, \theta)\right] .
$$

uniformly in $\sigma \in] 0,1]$.

ii) For fixed $\left.(\sigma, \mu) \in \mathbb{R}^{+} \times\right] 0,1\left[\right.$ and $0<\theta_{1}<\theta_{2}$, there exists $\bar{\lambda}=$ $\bar{\lambda}\left(\sigma, \mu, \theta_{1}, \theta_{2}\right)$ such that the following holds

$$
V E_{2}\left(\sigma, \lambda, \mu, \theta_{1}\right)<V E_{2}\left(\sigma, \lambda, \mu, \theta_{2}\right), \quad \forall \lambda>\bar{\lambda} .
$$

Proof. By direct computation it is easy to prove that $\frac{\partial V E_{2}}{\partial \lambda}(\sigma, \lambda, \mu, \theta)$ is negative for any $\lambda \in] 0,+\infty[$ and for any $(\sigma, \mu, \theta) \in] 0,1\left[\times \mathbb{R}^{+} \times \mathbb{R}^{+}\right.$, and the first claim of our statement follows. Now we prove the second part of the theorem. If we set $A(\sigma, \lambda, \mu, \theta)=1-(1+\sigma \theta \lambda)^{-\frac{\mu}{\theta}}$, where $(\sigma, \lambda, \mu, \theta) \in$ ] $0,1\left[\times \mathbb{R}^{+} \times \mathbb{R}^{+} \times \mathbb{R}^{+}\right.$, we have that, for any fixed $\left.\bar{\sigma} \in\right] 0,1[$,

$$
\frac{\partial V E_{2}}{\partial \theta}(\bar{\sigma}, \lambda, \mu, \theta)<0
$$

if and only if

$$
\frac{\frac{\partial A}{\partial \theta}(\bar{\sigma}, \lambda, \mu, \theta)}{A(\bar{\sigma}, \lambda, \mu, \theta)}>\frac{\frac{\partial A}{\partial \theta}(1, \lambda, \mu, \theta)}{A(1, \lambda, \mu, \theta)}
$$

Then, if we define

$$
H(\sigma, \lambda, \mu, \theta)=\frac{\frac{\partial A}{\partial \theta}(\sigma, \lambda, \mu, \theta)}{A(\sigma, \lambda, \mu, \theta)}=\frac{\mu}{\theta^{2}} \frac{\left[\frac{\sigma \lambda \theta}{\sigma \lambda \theta+1}-\log (1+\sigma \lambda \theta)\right]}{(1+\sigma \lambda \theta)^{\frac{\mu}{\theta}}-1},
$$

to get (32) for a given triplet $(\lambda, \mu, \theta)$ it will be sufficient to show that $\sigma \rightarrow$ $H(\sigma, \lambda, \mu, \theta)$ is decreasing in the interval $[\bar{\sigma}, 1]$. We show that this property holds uniformly with respect to $\sigma \in] 0,1]$ for sufficiently small values of $\lambda$ that depend on $(\mu, \theta)$ and this implies $i$ ). First, by using the Taylor expansion in the variable $u=\sigma \lambda \theta$ at $u=0$ with $\mu$ fixed, we see that $H$ can be extended as an analytic function to $u=0$ and that $H(\sigma, \lambda, \mu, \theta)=-\frac{\sigma \lambda}{2}+o(\sigma \lambda \theta)$. As a consequence, there exists $\varepsilon=\varepsilon(\mu)>0$ such that, if $0<\sigma \lambda \theta<\varepsilon$ then

$$
\frac{\partial H(\sigma, \lambda, \mu, \theta)}{\partial \sigma}=-\frac{\lambda}{2}+o(\sigma \lambda \theta)<0
$$


for any $\sigma \in] 0,1]$. It follows that for any $(\mu, \theta) \in \mathbb{R}^{+} \times \mathbb{R}^{+}$we can find a small value of $\lambda:=\lambda(\mu, \theta)$ such that the inequality (33) holds uniformly for $\sigma \in] 0,1]$. Hence, the same is true for (31), and property $i$ ) is proved. We move now to the proof of $i i)$. Direct computation shows that

$$
\operatorname{sgn}\left(\frac{\partial H}{\partial \sigma}(\sigma, \lambda, \mu, \theta)\right)=\operatorname{sgn}(K(\sigma, \lambda, \mu, \theta))
$$

where

$$
K(\sigma, \lambda, \mu, \theta)=\frac{\sigma \lambda \theta}{\sigma \lambda \theta+1}\left[(1+\sigma \lambda \theta)^{-\frac{\mu}{\theta}}-1-\frac{\mu}{\theta}\right]+\frac{\mu}{\theta} \log (1+\sigma \lambda \theta) .
$$

Now, for any given $(\sigma, \mu, \theta) \in] 0,1] \times \mathbb{R}^{+} \times \mathbb{R}^{+}$we have that $K \rightarrow+\infty$ as $\lambda \rightarrow+\infty$. Then, being $K$ continuous, a standard compactness argument allows to conclude that fixed any $\bar{\sigma} \in] 0,1]$, there exists $\lambda_{1}=\lambda_{1}(\bar{\sigma}, \mu, \theta)$ such that $K(\sigma, \lambda, \mu, \theta)>0, \forall \sigma \in[\bar{\sigma}, 1], \lambda>\lambda_{1}(\bar{\sigma}, \mu, \theta)$.

Actually, given $\theta_{1}>0$ and $\theta_{2}>0$ with $\theta_{2}>\theta_{1}$, by the same argument one can conclude the existence of a $\bar{\lambda}=\bar{\lambda}\left(\bar{\sigma}, \mu, \theta_{1}, \theta_{2}\right)$ such that $K(\sigma, \lambda, \mu, \theta)>0, \forall \sigma \in[\bar{\sigma}, 1], \theta \in\left[\theta_{1}, \theta_{2}\right], \lambda>\bar{\lambda}$.

As a consequence, we have that $\sigma \rightarrow H(\sigma, \lambda, \mu, \theta)$ is increasing in the interval $[\bar{\sigma}, 1]$ for any $\theta \in\left[\theta_{1}, \theta_{2}\right]$ and any $\lambda>\bar{\lambda}$, so that $\frac{\partial V E_{2}}{\partial \theta}>0$ for $\sigma \in[\bar{\sigma}, 1]$, for any $\theta \in\left[\theta_{1}, \theta_{2}\right]$ and any $\lambda>\bar{\lambda}$.

We conclude that fixed any $\sigma, \theta_{1}<\theta_{2}$ and $\mu$, there exists $\bar{\lambda}=\bar{\lambda}\left(\sigma, \mu, \theta_{1}, \theta_{2}\right)$ such that $V E_{2}\left(\sigma, \mu, \lambda, \theta_{1}\right)<V E_{2}\left(\sigma, \mu, \lambda, \theta_{2}\right)$, for all $\lambda>\bar{\lambda}$, and $\left.i i\right)$ is proved.

An immediate consequence of the previous result is the following:

Corollary 4.1 Under the assumptions of Theorem 4.3, given $\theta_{1}$ and $\theta_{2}$ with $0<\theta_{1}<\theta_{2}$, for all $(\sigma, \mu)$ the curves $\lambda \rightarrow V E_{2}\left(\sigma, \mu, \lambda, \theta_{i}\right)$ switch their order for large $\lambda$.

Remark 1 In the proof of $i)$, the function $\lambda(\mu, \theta)$ for which $\frac{\partial H}{\partial \sigma}<0$ uniformly with respect to $\sigma \in] 0,1]$ can take small values. However, we can extend its values by taking as $\lambda=\lambda(\mu, \theta)$ the unique function defined implicitly by

$$
K(1, \lambda, \mu, \theta)=0,
$$

where $K$ is given by (35). This statement follows from two facts. Firstly, $\frac{\partial K}{\partial \lambda}>0$ for the points satisfying (36) and the existence of $\lambda$ follows from the 
implicit function theorem. Since $\frac{\partial K}{\partial \lambda}>0$ this function is also globally unique. Secondly, the function $\sigma \rightarrow K(\sigma, \lambda, \mu, \theta), \sigma \in[0,1]$, satisfies $K(0, \lambda, \mu, \theta)=$ 0 and

$$
\frac{\partial K}{\partial \sigma}=\frac{\lambda \mu(\sigma \lambda \mu-1)}{(1+\sigma \lambda \theta)^{2}}\left(1-(1+\sigma \lambda \theta)^{-\frac{\mu}{\theta}}\right)
$$

so that it is decreasing in the interval $\left[0, \frac{1}{\lambda \mu}\right]$ and it is increasing in the interval $\left[\frac{1}{\lambda \mu}, 1\right]$. Therefore, $K(\sigma, \lambda, \mu, \theta)<0$ whenever $K(1, \lambda, \mu, \theta)<0$. For any given $(\mu, \theta) \in \mathbb{R}^{+} \times \mathbb{R}^{+}$, one can check that $K(1, \lambda, \mu, \theta)<0$ for small $\lambda$, and, by definition of $\lambda(\mu, \theta)$, this inequality holds for $\lambda \in] 0, \lambda(\mu, \theta)[$. Then, by construction, $i)$ of Theorem 4.3 holds by taking as function $\lambda(\mu, \theta)$ the one defined implicitly by (36).

One important qualitative feature of this function is that it is increasing in $\theta$. In order to prove this we can use a two dimensional implicit plot. Namely, setting $x=\frac{\theta}{\mu}, y=\mu \lambda$, from (36) we get

$$
\tilde{K}(x, y)=\frac{x y}{1+x y}\left[(1+x y)^{-\frac{1}{x}}-1-\frac{1}{x}\right]+\frac{\log (1+x y)}{x}=0
$$

We have plotted the contour line $\tilde{K}=0$ and verified that this is an increasing function $x \rightarrow y(x)$, for $x>0$ (not shown). Then, by the uniqueness of the implicit function, we conclude $\lambda(\mu, \theta)=\frac{1}{\mu} y\left(\frac{\theta}{\mu}\right)$, and $\theta \rightarrow \lambda(\mu, \theta)$ is increasing.

\section{$5 \quad$ Redefining vaccine efficacy}

In line with the rationale that measured discrepancies in vaccine protection arise from differences in frequency of exposure across settings, the identification of a consistent measure might require disentangling efficacy from exposure [3,10]. We have presented a mathematical framework for obtaining such measure. We propose the fitting of heterogeneous linear models, such as those in Section 4 (solid curves in Figure 3), to datasets from multi-center clinical trials, as introduced in Section 3 for tuberculosis [6] and malaria [11], to estimate $1-\sigma$, which is, effectively, a measure of efficacy per unit of exposure. The distributions of disease risk among individuals within trial sites would be simultaneously estimated.

It may be argued that currently used measures, based on the homogeneous models in Section 2, are more informative of the actual effect of the vaccine in each setting. We are not proposing that such analyses should be 

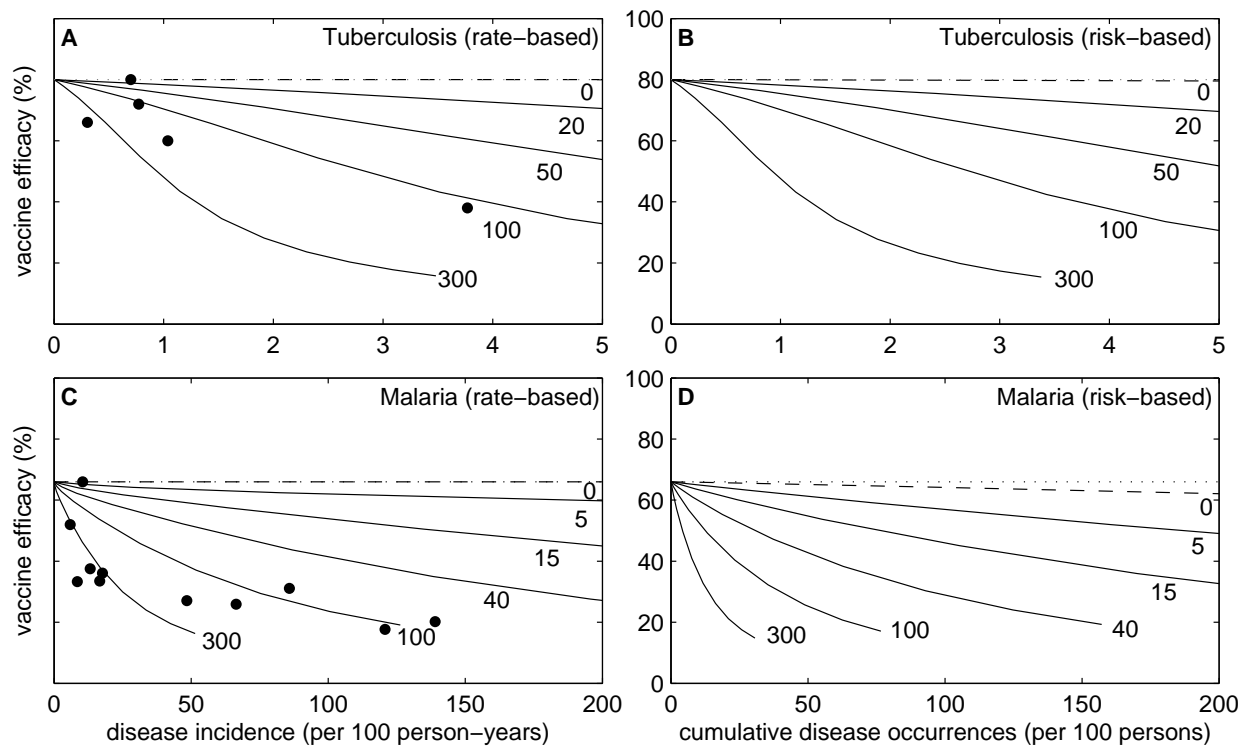

Figure 3: Vaccine efficacy on disease incidence gradients. A, Tuberculosis, data from [6], curves generated with formula (23) and $\gamma=0$. B, Tuberculosis, curves generated with formula (28) and $\gamma=0$. C, Malaria, data from [11], curves generated with formula (23) and $\gamma=12$. D, Malaria, curves generated with formula (28) and $\gamma=12$. Data points are countries where a trial has been conducted to estimate vaccine efficacy. Curves are labeled by variance in the risk distribution $q(x)$.

discarded, rather that they should not be called "vaccine efficacy??. Indeed our procedure relies on a collection of many such measurements across settings to inform the estimation of a more basic quantity, which has a clear interpretation and can be used in a variety of models for prediction in different contexts.

\section{Discussion}

As we have seen, despite the overwhelming evidence that vaccine efficacy, as estimated in clinical trials, decays with disease incidence, it is feasible to conceive a vaccine efficacy parameter that is incidence-independent and a model that reproduces the incidence-dependent observations when adequately parametrised. Such universal efficacy parameter can be estimated by fitting the model to vaccine efficacy data on a gradient of incidences. Ad- 
justing models to global data requires flexible risk distributions that can also be estimated in this process $[2,12]$. By this procedure, an efficacy measure can be obtained for any vaccine in a way that is globally consistent with trial outcomes when local distributions of individual risk are adequately included as an auxiliary component of the model.

Practical measures of vaccine efficacy are commonly based on rate ratios or risk ratios [13]. For illustration we have chosen two vaccines that have undergone randomised controlled trials in different locations and rate-based efficacy measures were provided (BCG against pulmonary tuberculosis [6] and RTS,S against malaria [11]). We have superposed a family of curves (parametrised by variance in the distribution of individual disease risk) with previously published data demonstrating that the curves describe the trends of interest as intuitively explained (Figure 3). Curves generated by ratebased or risk-based procedure are visually very similar.

Efficacy curves appear to satisfy an order such that the higher the variance in disease risk the lower the vaccine efficacy. Although this ordering is proved to break down for high enough forces of infection, numerical solutions indicate that this is unlikely to have practical relevance. In this sense, combating risk heterogeneity, by means of social protection, biological interventions or environmental control, might be considered as strategies to increase the benefits of vaccines.

In this paper we have considered individual heterogeneity in disease risk $[9,10]$, in contrast to other studies that have considered variation in the effect of the vaccine itself $[3,4,13]$. Although both distributions are likely to affect current measures of vaccine efficacy [16], we wanted to counter a perceived tendency in the research community to explain the observations entirely in light of the latter. Meanwhile, the full parameterisation of vaccine efficacy models remains an important subject for future research.

Acknowledgments: We thank two anonymous reviewers for constructive comments. MGMG is grateful to the Mathematical Biosciences Institute, Ohio State University, particularly Marty Golubitsky, for hosting during the development of these ideas.

\section{References}

[1] Diekmann O, Heesterbeek JAP (2000) Mathematical epidemiology of infectious diseases: Model building, analysis and interpretation. Wiley. 
[2] Gomes MGM, Aguas R, Lopes JS, et al (2012) How host selection governs tuberculosis reinfection. Proc R Soc B 279: 2473-8.

[3] Gomes MGM, Lipsitch M, Wargo AR, et al (2014) A missing dimension in measures of vaccination impacts. PLOS Pathog 10: e1003849.

[4] Halloran ME, Longini Jr IM, Struchiner CJ (2010) Design and Analysis of Vaccine Studies. Springer New York.

[5] Jiang V, Jiang B, Tate J, Parashar UD, Patel MM (2010) Performance of rotavirus vaccines in developed and developing countries. Hum Vaccin 6: $532-42$.

[6] Mangtani P, Abubakar I, Ariti C, et al (2014) Protection by BCG vaccine against tuberculosis: a systematic review of randomized controlled trials. Clin Infect Dis 58: 470-80.

[7] Margheri A, Rebelo C, Gomes MGM (2015) On the correlation between variance in individual susceptibilities and infection prevalence in populations. J Math Biol 71: 1643-1661.

[8] Miller JC, Volz EM (2013) Model hierarchies in edge-based compartmental modeling for infectious disease spread. J Math Biol 67: 869-899.

[9] O'Hagan JJ, Hernán MA, Walensky RP, Lipsitch M (2012) Apparent declining efficacy in randomized trials: Examples of the RV144 HIV vaccine and CAPRISA 004 microbicide trials. AIDS 26(2): 123-6.

[10] O'Hagan JJ, Lipsitch M, Hernán MA (2014) Estimating the perexposure effect of infectious disease interventions. Epidemiol 25(1): 1348.

[11] The RTS,S Clinical Trials Partnership (2015) A phase 3 trial of RTS,S/AS01 malaria vaccine in African infants. N Engl J Med 367: 2284-95.

[12] Smith DL, Dushoff J, Snow RW, Hay SI (2005) The entomological inoculation rate and its relation to the prevalence of Plasmodium falciparum infection in African children. Nature 438: 492-5.

[13] Smith PG, Rodrigues LC, Fine PEM (1984) Assessment of the protective efficacy of vaccines against common diseases using case-control and cohort studies. Int J Epidemiol 13: 87-93. 
[14] Vaupel JW, Manton KG, Stallard E (1979) Impact of heterogeneity in individual frailty on the dynamics of mortality. Demography 16: 439454.

[15] Vaupel JW, Yashin AI (1985) Heterogeneity's Ruses: Some surprising effects of selection on population dynamics. Am Stat 39: 176-85.

[16] White MT, Griffin JT, Drakeley CJ, Ghani AC (2010) Heterogeneity in malaria exposure and vaccine response: implications for the interpretation of vaccine efficacy trials. Malar J 9: 82. 